\title{
BILATERAL INTERSCALENE BRACHIAL PLEXUS BLOCK FOR SIMULTANEOUS BILATERAL UPPER EXTREMITY SURGERY
}

Mihran Portugalyan 1, André Delgado 1

1- Serviço de Anestesiologia, Reanimação e Dor, Hospital Professor Doutor Fernando Fonseca

\section{BACKGROUND AND AIMS}

Bilateral brachial plexus blocks are rarely performed in modern anaesthesia practice due to concerns about local anesthetic toxicity, bilateral diaphragmatic paralysis and pneumothorax. However, with the use of ultrasound guidance and by spacing blocks apart in time, it may be considered in selected patients. We report a clinical scenario where this approach seemed appropriate.

\section{METHODS}

Patient: Female, 58 yo, ASA II. Predicted difficult airway.

Physical examination: Severe retrognatism. Upper Lip Bite Test: Class III. Mallampati Score: Class III.

Procedure: Bilateral shoulder arthroplasty.

Technique: Left interscalene brachial plexus block was performed under direct echographic visualization, injecting $15 c c$ of $0,75 \%$ Ropivacain. After 120 minutes the arthroplasty was completed and the block was repeated contralaterally. Total Ropivacain: $225 \mathrm{mg}(2,8 \mathrm{mg} / \mathrm{Kg})$.

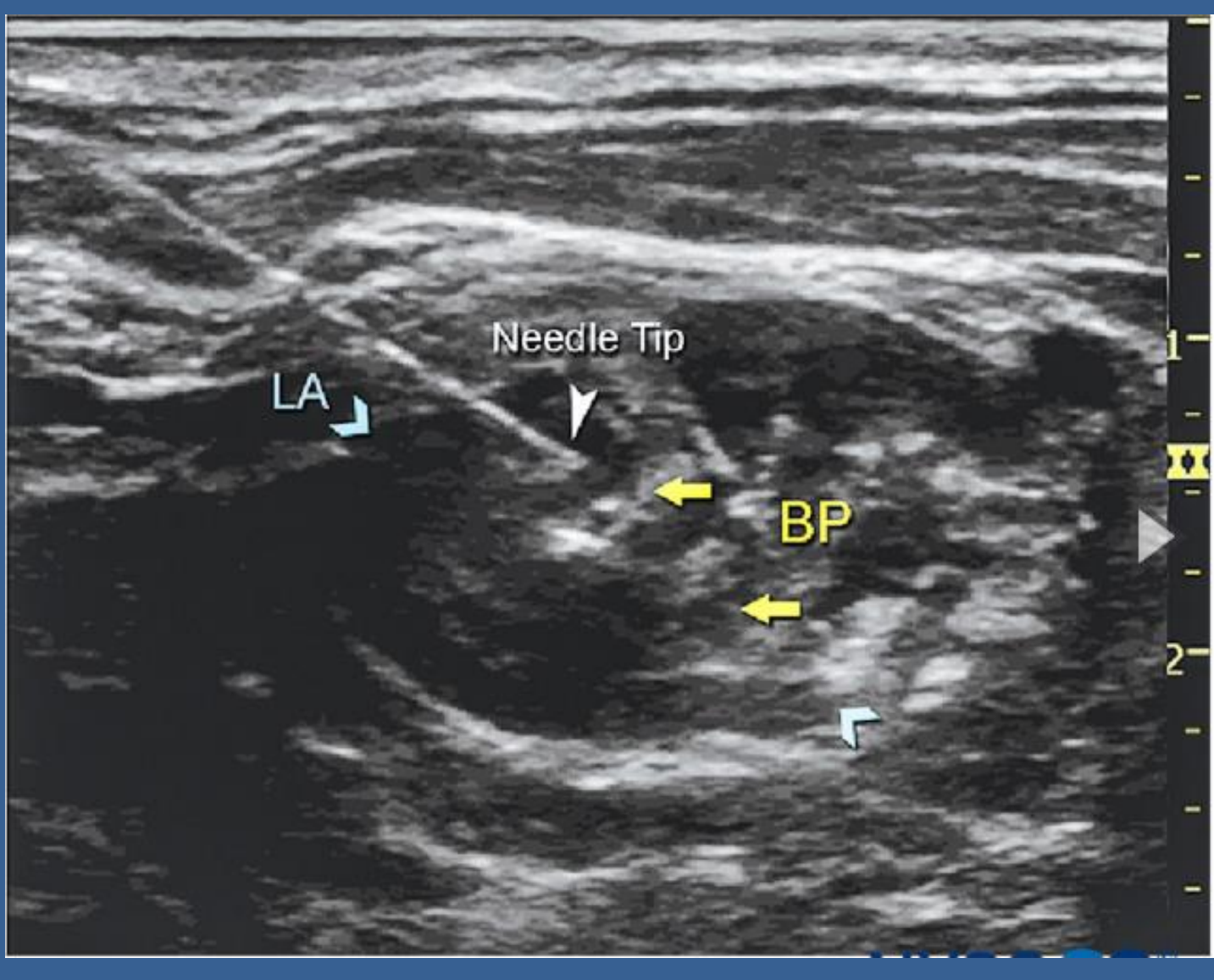

Interscalene plexus block (Fig.1): Echographic visualization depicting a needle (white arrowhead) placement in the interscalene groove, with dispersion of local anaesthetic (LA) surrounding the brachial plexus (BP).

\section{RESULTS}

Both blocks worked adequately with minimal patient discomfort, without difficulty breathing and with stable vital signs during and after surgery.

\section{CONCLUSIONS}

Bilateral brachial plexus blocks should be considered for simultaneous bilateral surgery when the risks of a general anaesthesia outweigh its beneficts (low cardiovascular function or a predicted difficult airway), provided adequate pulmonary reserve is present. Ultrasound guidance can reduce the volume of local anaesthetic and the rate of phrenic nerve involvement. Spacing blocks apart in time helps to detect evolving signs of toxicity from the first block and ensures that peak absoption does not occur simultaneously. In this case report we found this technique to be effective and safe, with the added advantage of ensuring a prolonged postoperative analgesia. 